\title{
ELIAS CANETTI E TONI MARAINI A MARRAKECH: UN VIAGGIO NELLA MEMORIA
}

\author{
ELIAS CANETTI AND TONI MARAINI: A JOURNEY INTO MEMORY
}

Mahmoud Jaran

University of Jordan

\section{RESUMEN:}

Nel presente articolo, si prendono in esame due opere di due autori decisamente lontani l'uno dall'altra, ma che si incontrano negli stessi luoghi nei loro sentimenti. La prima opera è "Le voci di Marrakech" (1968), dello scrittore bulgaro di famiglia ebrea e premio Nobel per la Letteratura, Elias Canetti, mentre la seconda è "Ultimo tè a Marrakech", un diario di viaggio uscito nel 2000, della scrittrice e poetessa italiana, Toni Maraini.

Entrambi questi viaggi nel mondo arabo, sono ritratto socioculturale del Marocco contemporaneo, conditi da squarci di vita quotidiana, i quali in modi e forme differenti, offrono la possibilità di compiere una serie di analisi e di riflessioni che interessano la tesa relazione ontologica tra occidente $\mathrm{e}$ oriente, ma anche una vasta gamma di campi interdisciplinari: dal femminismo alla teoria postcoloniale; dalla politica ai cultural studies.

\section{PALABRAS ClAVE:}

opere, Elias Canetti, Toni Maraini, postcoloniale, femminismo

\section{ABSTRACT:}

In this article, we examine two works by two authors who are very distant from each other, but who meet in the same places in their feelings. The first work is "The voices of Marrakech" (1968), by the Bulgarian writer of Jewish family and Nobel Prize for Literature, Elias Canetti, while the second is "Last tea in Marrakech", a travel diary published in 2000, by Italian writer and poet, Toni Maraini.

Both of these trips to the Arab world are sociocultural portraits of contemporary Morocco, seasoned with glimpses of everyday life, which in different ways and forms, offer the possibility of carrying out a series of analyzes and reflections that affect the tense ontological relationship between the West and east, but also a wide range of interdisciplinary fields: from feminism to postcolonial theory; from politics to cultural studies.

\section{KEYWORDS:}

works, Elias Canetti, Toni Maraini, postcolonial, feminism 
Raccontare una città, nella letteratura odeporica ${ }^{1}$, presuppone comunemente una descrizione di ambienti, colori, odori e, possibilmente, dei suoi abitanti, della loro quotidianità fondata su principi socio-economici, culturali e linguistici. L'obiettivo dello scrittore, in questo senso, è quello di cogliere il legame che intercorre tra il passato e la modernità, ossia di ricercare quella catena spazio-temporale che determina i confini della memoria. Per cogliere tale legame, una volta nella città straniera, lo scrittore si serve di alcuni elementi quali: la questione dell'identità, l'indagine etnologica ed il teso rapporto che si instaura tra il soggetto narrante e l'oggetto narrato. Le due opere che si prenderanno in esame in questa sede dimostrano come la città divenga uno «spazio inter-medio», tanto per dirla con il critico indiano Homi Bhabha, dove l'identità ibrida del personaggio/narratore è in continua trasformazione ${ }^{2}$. Si tratta di due autori decisamente lontani l'uno dall'altra, ma che si incontreranno negli stessi luoghi nei loro sentimenti: lo scrittore bulgaro di famiglia ebrea e premio Nobel per la Letteratura Elias Canetti; e Toni Maraini, una scrittrice italiana, femminista e studiosa del mondo maghrebino. L'approccio narrativo di entrambi al Marocco, in particolar modo a Marrakech e dintorni, non solo riscontra spazi inter-medi, ma li spinge fino a celare la propria identità.

Fondata nell'epoca della dinastia almoravide (verso il 1070), Marrakech divenne sotto il regno degli Almohadi (1147-1269) un crocevia commerciale ed un ricco centro di cultura, di filosofia e di architettura. La prosperità della città marocchina, capitale delle due dinastie, tuttavia non durò a lungo: con l'arrivo dei Merinidi al potere nel Nord Africa (1269-1465), Marrakech dovette infatti cedere il prestigio della capitale alla «città rivale» Fes. All'inizio del XVI secolo, Marrakech passò alla dinastia Sadiana che la scelse come capitale, dandole una vera rinascita culturale e architettonica. Da allora, l'alternanza di fasi di regresso e progresso interesserà la città fino ai giorni nostri: dal governo degli Alawidi, che fecero Meknes capitale, all'arrivo dei colonizzatori europei nel XIX secolo, epoca che rivide Marrakech centro di traffici commerciali, fino all'Indipendenza, ottenuta nel 1956, quando la sua popolazione aumentò enormemente, divenendo nei decenni successivi un'apprezzata meta per i turisti occidentali.

Le varie dinastie che governarono Marrakech non misero mai confini alla sua multietnicità: arabi, berberi, ebrei, europei ed africani subsahariani. Diversi accenni alla sua condizione multietnica sono presenti ne Le voci di Marrakech (1968) di Elias Canetti. Il diario di viaggio, che racconta il breve soggiorno di Canetti a Marrakech nel 1954, offre un affresco seminarrativo affollato da commercianti arabi, berberi, personaggi europei e da una famiglia ebrea indigena. Il primo ostacolo che incontra il romanziere bulgaro nella città marocchina è la lingua. Ciò non è dovuto al fatto che la mancanza di padronanza della lingua araba possa impedire la comunicazione con gli indigeni (egli usa infatti il francese come lingua franca quando si rivolge a questi³), ma perché il 
linguaggio, come sostiene Iain Chambers, è il primo fattore ad ispezionare la memoria (Chambers 2003: 128). Da qui nasce l'idea canettiana di intraprendere, come prima tappa del viaggio, un'indagine linguistica atta a decodificare le emozioni, i desideri e le repressioni che costituiscono la memoria della città.

Già il titolo del diario, che contiene la parola "voce», suggerisce tale intento dell'autore $^{4}$, così come lo suggeriscono altresì alcuni titoli dei capitoli. Si pensi alla serie di capitoli consecutivi, dal terzo al sesto, intitolati «Le grida dei ciechi», «La saliva del marabutto», «Casa silenziosa e tetti deserti». All'inizio de Le grida dei ciechi, Canetti rivela difatti la problematica linguistica, mettendola in relazione con la sua inesprimibilità dovuta alla distinzione che il racconto tenta di operare tra lingua e linguaggio:

Tento di raccontare qualcosa, ma subito ammutolisco e mi accorgo di non aver detto ancora niente. Una sostanza meravigliosamente lucente che non riesce a fluire rimane dentro di me e si fa beffe delle parole. Sarà per la lingua, che là non capivo e che ora, a poco a poco, deve tradursi in me? Si trattò di avvenimenti, immagini, suoni, il cui senso si formò allora, ma che non furono percepiti né definiti per mezzo delle parole, stanno al di là delle parole, e sono più profondi e più ambigui delle parole. (Canetti 2000: 27)

I ciechi che mendicano in piazza ripetendo eternamente il nome di Allah, unica parola araba che l'autore conosce, conducono Canetti a riflettere sul significato dei suoni «mille volte più impressionanti di quelli visivi» (Canetti 2000: 27), ed il ruolo che possano avere nell'esperienza mistica ${ }^{5}$. Tale esperienza si intensifica ulteriormente nel capitolo successivo quando Canetti incontra un mendicante marabutto intento ad assaggiare le monete che riceve dai passanti. Nonostante l'immensa tentazione di liquidare il fatto in una «nota esotica» (Canetti 2000: 35), l'autore percepisce che si tratta, invece, di una forma di linguaggio che il vecchio santone pratica per benedire $\mathrm{i}$ donatori.

Dal tetto della casa silenziosa dove alloggia, Canetti vorrebbe volentieri scoprire la realtà celata della città araba, ossia il mondo femminile. L'autore svela questo intento, tipico della tradizione orientalistica ${ }^{6}$, a più riprese nel diario. Già nel secondo capitolo Canetti trova affascinante la velata sfera femminile orientale: «In una società che tiene nascosto così tanto di sé, che agli stranieri cela gelosamente l'interno delle sue case, la figura e il volto delle sue donne» (Canetti 2000: 23). L'indagine linguistica fa sì che tale aspirazione non si limiti soltanto ad un aspetto voyeuristico teso a soddisfare un panorama visivo fiabesco, ma s'estende fino ad includere altresì la dimensione dell'udito. «Qui, pensavo, vedrò delle donne come nelle favole, da qui potrò guardare nei cortili delle case vicine e ascoltare di nascosto il loro affaccendarsi» (Canetti 2000: 41). 
Non passerà molto prima che questo desiderio venga esaudito. Nel quinto capitolo «La donna della grata», la ricerca del linguaggio e l'esperienza mistica raggiungono l'apice, grazie all'incontro tra Canetti ed una sconosciuta marocchina, la quale s'affaccia dalla finestra della propria casa e gli rivolge la parola: «Disse molte frasi che scorrevano leggere, e ognuna di quelle frasi era fatta di parole carezzevoli» (Canetti 2000: 43). Lo scrittore, conquistato dal fascino del volto senza velo e soprattutto dall'»effetto sonoro» proveniente dalla finestra, prova una sensazione di carattere mistico, quasi religioso. Non a caso Canetti descrive quella donna come «un miracolo, un'apparizione» (Canetti 2000: 45)7 . Benché l'autore non comprenda le parole della donna della grata e dei mendicanti ciechi, vi rimane un valore inestimabile per l'ignoto, l'ambiguo, il «gioco degli occhi». Ecco perché le pagine dedicate alle situazioni extralinguistiche superano quantitativamente e qualitativamente quelle delle scene dialogate e dell'ordinaria comunicazione in francese. È il linguaggio che prevale sulla lingua ${ }^{8}$.

I capitoli centrali (VII e VIII) sono dedicati alla comunità ebraica di Marrakech. Tale posizione centrale non rispetta necessariamente la cronologia del diario. Almeno due ragioni spingono a credere che questi capitoli siano volutamente disposti in una posizione centrale nell'opera di Canetti: la prima concerne lo stretto rapporto che lega lo scrittore con il quartiere ebraico (Mellah) di Marrakech, la piazza principale del quale viene denominata da lui «il cuore» (Canetti 2000: 67); la seconda riguarda, invece, la stessa comunità ebraica, descritta da Canetti in termini socioculturali come se fosse una via di mezzo tra gli indigeni orientali e gli europei. È vero che alcuni ebrei stanno nei suk «distesi pigramente come gli arabi» (Canetti 2000: 53), ma essi sono «vestiti all'europea» (Canetti 2000: 51).

La piazza battezzata «il cuore» diventa per Canetti il luogo della memoria par excellence. I riferimenti impliciti alle proprie remote origini spagnole e all'espulsione degli ebrei dalla penisola iberica nel 1492 consentono all'autore bulgaro di confidare al lettore non solo lo speciale affetto che egli conserva per questa piazza: «Da lì non volevo più andarmene, ci ero già stato centinaia di anni prima, ma lo avevo dimenticato, ed ecco che ora tutto ritornava in me» (Canetti 2000: 57), ma anche un alto senso di immedesimazione con il luogo della memoria che coincide con il luogo della storia: «io ero quella piazza. Credo di essere sempre quella piazza» (Canetti 2000: 57). L'assimilazione con l'altrove e con l'altro è ulteriormente intensificata quando Canetti svela l'unico nome indigeno del suo viaggio nella città: Elie, un giovane marocchino ebreo che accoglie lo scrittore a casa sua. I nomi somiglianti, Elias e Elie, e le origini identiche - entrambi ebrei sefarditi - rendono l'incontro piacevole e familiare. Da quel momento il viaggiatore bulgaro comincia a vedere la terra straniera e la sua gente come se si stesse guardando allo specchio. 
Prevedibilmente, gli accenni alla comunità ebraica di Marrakech ne Le voci riguardano da vicino il vivere quotidiano degli ebrei nel bazar della Mellah. Se la varietà dei loro tratti fisionomici rappresenta il primo aspetto che un turista può notare, ciò non impedisce a Canetti di scorgere una caratteristica psicologica che li rende, in qualche modo, omogenei:

Comunque avevano tutti qualcosa in comune, e appena mi fui abituato alla grande varietà dei loro volti e delle loro espressioni, cercai di scoprire in che cosa realmente consistesse questo tratto comune. Avevano una speciale rapidità nell'alzare lo sguardo e nel farsi un'opinione sulla persona che camminava davanti a loro. Non una sola volta mi capitò di passare inosservato. (Canetti 2000: 52)

Non sorprendono la precisione descrittiva di un autore quale Canetti, né l'atteggiamento dei commercianti ebrei nei suoi confronti. D'altronde, benché agli ebrei fu conferita uguaglianza al pari dei musulmani nel Marocco postcoloniale, la minoranza ebraica, ed in particolar modo quella fetta appartenente alla classe borghese, si trovavano in uno stato incerto che oscillava tra il timore di essere discriminati e l'ansia di perdere alcuni previlegi concessi dalle autorità locali sia nel settore pubblico, sia in quello privato. Un autentico profilo sulla comunità ebraica borghese nell'era postcoloniale viene offerto dal libro Morocco - Independant, uscito nel 1961, di Rom Landau. L'arabista inglese, d'origine polacca, scrive:

Il Marocco è l'unico Paese nel mondo arabo dove gli ebrei potevano vivere senza essere mai costretti a lasciarlo. Sotto le autorità islamiche, per mille e duecento anni, fu permesso loro di praticare le loro preghiere e conservare le loro tradizioni ed i loro costumi. A differenza della maggior parte degli stati europei, in Marocco non emerse mai alcuna campagna antisemita. Vi furono nel passato alcuni casi di discriminazione, ma dall'Indipendenza in poi, gli ebrei vennero trattati al pari dei musulmani. Alcuni occuparono posizioni importanti nel governo. In ambito amministrativo, il numero degli ebrei che lavoravano come segretari e interpreti superava quello dei musulmani. Ciò è comprensibile, poiché la borghesia ebraica, nota per l'intelligenza istintiva, per il lavoro duro e per l'anelito all'istruzione, assicurava alle autorità un modello esemplare di funzionari. (Landau 1961: 238239)

Un altro luogo che coglie nel segno la sensibilità di Canetti è Djema el Fna. L'origine del nome di questa piazza, situata nel centro della medina, è incerta. Secondo le fonti ufficiali dell’UNESCO, la quale ha nominato la piazza «Patrimonio orale e immateriale dell'umanità» nel 2001 grazie alla presentazione della sua candidatura da parte dello scrittore spagnolo Juan Goytisolo, il nome deriva dalla «moschea dell'annientamento» (Borghi 2006: 3). «Djema», infatti, significa «moschea», ma anche più semplicemente, «raduno»; quanto, invece, al termine «Fna», questo può riferirsi al concetto della «morte» (è, quindi, il posto dove si svolgono le pubbliche esecuzioni), alla «distruzione» 
(in riferimento ad una moschea distrutta in epoca almoravide), oppure ad un «cortile esterno» (un largo spiazzo fuori dalla moschea) ${ }^{9}$.

In questa piazza si svolge gran parte della vita socioculturale dei cittadini di Marrakech. Se, come afferma Canetti nella sua opera monumentale Massa e potere (1960), i musulmani si riuniscono in quattro precise occasioni ${ }^{10}$, Djema el Fna rappresenta un'altra circostanza spazio-temporale di raduno per eccellenza. In questa piazza giungono ogni giorno venditori d'acqua, acrobati, ammaestratori di serpenti, dottori, dentisti, cantastorie. A quest'ultimi Canetti dedica un capitolo ne Le voci dal titolo "Cantastorie e scrivani», in cui emerge un' interessante riflessione metaletteraria, tipicamente canettiana ${ }^{11}$, che mette a confronto la letteratura «di carta» e la tradizione orale. Appartenente alla prima categoria, lo scrittore bulgaro non solo ammette la superiorità dei cantastorie: «mi apparvero come fratelli maggiori e migliori di me» (Canetti 2000: 95), ma addirittura prova disprezzo per chi vive di letteratura, protetto da tavoli, porte e carta. Considerare i cantastorie come «fratelli maggiori» trova una sua giustificazione stilistico-estetica nell'ambito della storia della letteratura. Essi rappresentano per Canetti quell'elemento letterario che meglio garantisce la stretta relazione tra il passato e la modernità. Non a caso l'autore li vede come «un'enclave di vita antica, di vita intatta» (Canetti 2000: 95), come se fossero gli aedi omerici, i hakawati de Le mille e una notte, i cantori medievali, oppure i griot dell'Africa occidentale ${ }^{12}$.

La «vita antica» e «intatta» dei cantastorie marocchini in piazza va di pari passo con la realtà, storicamente statica, che contraddistingue il commercio nel suk. Canetti osserva, a tal proposito, il contrasto tra la modernità (delle merci) ed il passato, rilevato nella forma antica di presentare le merci ${ }^{13}$. Il giudizio di Canetti, ciononostante, non va colto come facente parte di quella visione orientalistica che ribadisce, secondo Edward Said, l'immutabilità dell'oriente ${ }^{14}$. Si tratta, piuttosto, di un'osservazione più vicina alla concezione riguardante l'aspetto «preistorico» delle società pre-consumistiche che troviamo, ad esempio, nelle opere terzomondiste del poeta-regista italiano Pier Paolo Pasolini ${ }^{15}$. Canetti, come Pasolini, predilige l'antichità nelle relazioni sociali: ciò pare evidente nella distinzione che egli opera tra le leggi del suk marocchino ed il mercato europeo, tra il commercio come «arte antichissima» (Canetti 2000: 26) e lo scambio che rispetta «il prezzo fisso», dove «qualsiasi imbecille riesce a trovare le cose di cui ha bisogno» (Canetti 2000: 95).

Lo scontro tra tradizione e modernità nella Marrakech di Canetti, dunque, rompe l'armonia con il filone orientalistico che vede nell'oriente un mito. Lo scrittore bulgaro affronta questa tematica con uno sguardo realistico che dipinge la società marocchina, liberandola da comodi stereotipi e pregiudizi affrettati.

Nel capitolo intitolato «Shahrazad» (nome che rievoca il celebre mito de Le mille e una notte, ma ne Le voci si riferisce semplicemente ad un locale francese nella città vecchiadi 
Marrakech), Canetti illustra un aspetto fondamentale della città: il multietnicismo. Il bar francese è frequentato principalmente da francesi, americani, inglesi ed arabi: un nucleo multietnico in cui s'incontrano e si scontrano razze, lingue e religioni diverse. La modernità del locale, così come viene descritta da Canetti, si contrappone all'antichità delle piazze di Marrakech. Se in «Shahrazad» vige un'atmosfera di razzismo e intolleranza dettata da norme capitalistiche e da una «visione moderna» che custodisce il locale entro coordinate spazio-temporali ben precise, le piazze del centro storico, con la loro condizione che va oltre il tempo e lo spazio, rimangono, per Canetti, un modello esemplare di convivenza sociale e tolleranza. Nel bar francese si sussurrano storielle di matrimoni misti falliti, aneddoti fondati su pregiudizi razziali e notizie su arabi incivili e americani che vanno accolti con lo sfollagente (Canetti 2000: 121).

La presenza degli arabi in «Shahrazad» permette all'autore di tornare ancora una volta al conflitto tra modernità e tradizione nella società conservatrice di Marrakech. Egli scrive: "C'erano anche degli arabi i quali, però, erano vestiti all'europea oppure bevevano, il che bastava, ai loro occhi almeno, a renderli moderni e europei» (Canetti 2000: 112). Tale valutazione oggettiva (che si può scorgere nella frase «ai loro occhi») introduce inoltre un atteggiamento dell'autore che respinge tutti gli stereotipi esposti dalla signora franco-cinese Mignon, padrona del locale, la quale considerava «barbari» gli indigeni e gli americani. Canetti commenta questo comportamento così: «non ho mai trovato in nessuno pregiudizi così primitivi e incrollabili come in quella donna» (Canetti 2000: 113).

L'atteggiamento antirazzista di Canetti non sorprende i lettori, dato che la ricchezza contenutistica del viaggio a Marrakech è identificabile proprio nella ricerca di incontri con il maggior numero possibile di persone appartenenti a razze diverse ed a vari ceti sociali: arabi poveri (mendicanti) e arabi ricchi (frequentatori di Shahrazad), berberi, ebrei, francesi, italiani (Ginette), ecc. A tutti questi, però, Canetti cela le sue vere origini, limitandosi, per comodità, a dire di essere «inglese». Lo scrittore bulgaro, naturalizzato britannico, spiega questa scelta in questi termini: ««Dall'Inghilterra,» dissi «da Londra». Per non confondere la gente, mi ero abituato a semplificare così la mia risposta» (Canetti 2000: 71). Il vero motivo che induce l'autore a lasciare passare la questione della propria identit geografica, tuttavia, si potrebbe individuare nel fatto che Canetti, in questa sua indagine etnologica, non si considera un vero protagonista. I veri protagonisti sono gli abitanti del luogo, i loro costumi, le piazze di Marrakech (non a caso l'ultimo capitolo è dedicato alla grande piazza centrale), e soprattutto i suoni emessi dagli angoli più lontani di queste piazze. Notando un mendicante che si esprime soltanto con un lungo «a-a-a-a-a», lo scrittore conclude il libro ed il suo viaggio a Marrakech con queste parole: 
Forse non aveva la lingua per formare la «1» di « Allah», e il nome di Dio si accorciava per lui in «a-a-a-a-a». Ma era vivo, ed emetteva il suo unico suono con uno zelo e una costanza senza pari, lo emetteva per ore e ore fino a quando, nella piazza immensa, non restava che un unico suono, il suono che sopravviveva a tutti gli altri suoni. (Canetti 2000: 126)

Il viaggio nella memoria spazio-temporale ed il rapporto conflittuale tra tradizione e modernità in terra marocchina sono temi presenti anche in Ultimo tè a Marrakech, un diario di viaggio, uscito nel 2000, della scrittrice e poetessa italiana, Toni Maraini. Si tratta di una raccolta di quattordici racconti ${ }^{16}$ che offrono un ritratto socioculturale del Marocco contemporaneo, condito da squarci di vita quotidiana e da alcuni momenti narrativi che oscillano tra il dialogo (quasi sempre con «gente del posto») e il monologo. Oltre al suo valore letterario, la raccolta si presenta come un'indagine sociologica colma di analisi e riflessioni che possono interessare una vasta gamma di campi interdisciplinari: dal femminismo alla teoria postcoloniale; e dalla politica ai cultural studies.

Se il viaggio di Canetti a Marrakech è stato breve, quello di Maraini in Marocco è durato trentatré anni, di cui, come riportato nell'omonimo racconto «Ultimo tè a Marrakech» ${ }^{17}$, vent'anni nella piazza Djema el Fna e dintorni (Maraini 2000: 79). Il lungo soggiorno della scrittrice italiana in Marocco non spiega solo la profonda conoscenza della realtà sociale, politica e culturale del paese maghrebino, ma serve anche ad approfondire alcuni temi che ne Le Voci di Canetti erano solo stati accennati. Tra questi spiccano argomenti inevitabili quali la questione femminile, il cammino verso la modernità, il razzismo e la tesa relazione ontologica tra occidente e oriente.

Maraini è così attenta alla questione della donna magrebina da dedicarle racconti interi che vedono la presenza di personaggi esclusivamente femminili. In questi racconti (si pensi a «Una giornata, un fiume»; «L'ultimo pane»; «L'orologio cosmico») si potrebbe cogliere un'acuta visione femminista che copre un'interessante varietà di temi concernenti alcune piccole realtà matriarcali, i costumi, la condizione femminile che vacilla tra vincoli religiosi ed altri tradizionali, e la donna marocchina nel mondo simbolico. Se in Canetti il volto femminile marocchino è gelosamente nascosto agli stranieri per motivi religiosi, nei racconti di Maraini questo fatto viene esposto in un modo assai più esplicativo. Nel caso di Fatima, protagonista del racconto «Una giornata, un fiume», ad esempio, la questione del velo islamico viene presentata in chiave popolare basata sul costume: «Fatima non scrive; non parla francese benché conosca lo spagnolo, porta la gillaba e, quando esce, si mette sul volto una veletta; non è un obbligo ma un'usanza, un modo di vestirsi» (Maraini 2000: 31). Più curioso ancora è il rapporto tra la figura femminile e la simbologia di alcuni prodotti artigianali tradizionali come il pane della Grande Festa18, il quale «racchiude un compendio di 
concetti non verbali, plastici e semantici. Contiene i dati di una antica simbologia» (Maraini 2000: 53). Le donne che fanno il pane della Grande Festa ${ }^{18}$ probabilmente non conoscono i dettagli simbolici che stanno al centro di quest'»arte»; esse, tuttavia, non cambierebbero «mai una procedura e usanza che perpetua come inalienabile e bella» (Maraini 2000: 56).

Ma se le connotazioni simboliche non mutano nel tempo, la società marocchina, con la sua componente femminile, è più soggetta al cambiamento. Questo conduce ad una differenza tematica tra l'opera di Canetti, che descrive la società marocchina in termini statici, per dirla con de Saussure sincronici ed i racconti di Maraini, che mettono in evidenza l'evoluzione del ruolo femminile nella stessa società raggiungendo una dimensione più diacronica. Una delle ragioni di questa differenza è ovviamente la durata del soggiorno di entrambi gli autori europei in Marocco.

È interessante notare, però, avvicinando queste due opere che, se negli anni Cinquanta era un fatto comune che un locale potesse essere gestito da una donna francese (Madame Mignon ne Le voci), in tempi più recenti, anche una donna indigena può svolgere una simile attività. La narratrice del racconto sopraccitato «Una giornata, un fiume» osserva: «Una donna tradizionale che gestisce un locale è una significativa novità» (Maraini 2000: 22) ${ }^{19}$. Non mancano nella raccolta, inoltre, vari riferimenti all'impegno di certi personaggi-donne nel campo femminista. È il caso di Fatima che denuncia fortemente "oscurantismi e fatalismi storici», e di 'Aisha, "tenace e agguerrita, munita di dati, cifre e meticolose statistiche pubblicati in saggi e libri di analisi e denuncia sulla condizione della donna e dell'infanzia in Marocco» (Maraini 2000: 61-62).

Lo sfondo analitico del mutamento della sfera femminile nella società marocchina ci porta direttamente ad una tematica altamente cara alla scrittrice italiana, ossia il conflitto tra tradizione e modernità. L'autrice italiana, che tende qui a scartare in toto la prospettiva eurocentrica in questo dibattito ${ }^{20}$, preferisce lasciare la parola ad una certa classe di intellettuali marocchini: si tratta di un gruppo di artisti, scrittori e poeti che Maraini ha conosciuto durante il suo soggiorno in Marocco, ai quali l'autrice concede, nella raccolta, uno spazio narrativo piuttosto vasto. La disamina del punto di vista dell'»altro» in Ultimo tè a Marrakech rappresenta indubbiamente un passo avanti rispetto all'indagine «unilaterale» di Canetti, in quanto fornisce una visione ricca, ibrida ed eterogenea delle realtà storica, sociale, culturale e politica del Marocco. Oltre agli scrittori e pensatori affermati - conosciuti anche in occidente - quali Abdallah Laroui, Tahar Ben Jelloun e Mohammad Choukri, viene illustrato nei racconti il pensiero di un certo poeta dal nome Mustafà, il quale spiega il fallimento del cammino verso la modernità in Marocco così: 
Vi è stato fallimento delle politiche sociali e culturali, delle impostazioni ideologiche, delle borghesie mercantili, fallimento di un capitalismo da terzo mondo, di un populismo ottuso. Ma si addebitano all'idea di modernità anche fallimenti dovuti ad altre cause, a una modernizzazione frettolosa, per esempio, che non ha affrontato ignoranza, arcaismo e feudalesimo politico, insomma che, in realtà, non è «moderna». (Maraini 2000: 68)

A questa lucida osservazione di Mustafà, aggiunge Hamed, un altro intellettuale marocchino, una nota di fondamentale importanza che completa il quadro del dibattito sul rapporto tra il Marocco e la modernità: «Le si addebitano gli effetti di un mondialismo da neo-colonialismo e di una mancata riforma o giustizia nella modernità» (Maraini 2000: 68). La critica della politica socio-economica locale non può essere separata, insomma, dalla denuncia, lanciata dagli intellettuali, contro le nuove forme di colonialismo ed egemonia occidentale, responsabili dell'arretratezza del paese maghrebino. Che sia finita l'epoca del colonialismo o meno è un'argomentazione che sta al centro della disputa sulla modernità della società marocchina. Nel suo celebre volume L'histoire du Maghreb (1970), Abdallah Laroui commenta quest'atteggiamento, sostenendo che l'epoca coloniale è terminata: alcuni la difendono esplicitamente, altri in modo più implicito; c'è chi la accusa come se fosse l'origine di tutti i mali; e c'è chi la considera come una ricchezza per la natura e per l'individuo. [...] Elogiare quest'epoca con frasi negative o positive non si è fermato, probabilmente perché l'epoca coloniale non è ancora morta del tutto. (Laroui 1970: 378)

In tal senso, il colonialismo, per gli intellettuali marocchini, non rappresenta solo uno dei principali elementi che ostacolano il percorso verso la modernità, ma rappresenta anche un fattore storico-culturale che ha contribuito ad allargare il divario tra le sponde del Mediterraneo e, più generalmente, tra occidente e oriente in termini di rappresentazione. Gli stereotipi derivati dall'epoca coloniale ${ }^{21}$ e dalla post-Indipendenza sul mondo maghrebino vengono respinti tutti da Maraini: a partire dalla famosa formula orientalistica che vede nell'oriente un mondo irrazionale ${ }^{22}$, fino a clichés più recenti concernenti il marocchino immigrato, clandestino, ecc. (Maraini 2000: 166). È persino esplicita la volontà di Maraini di offrire ai suoi lettori un affresco culturale del Marocco, lontano da «abbellimenti» stereotipati e da ornamenti superflui, per non dire ingannevoli, tipici della letteratura orientalistica. L'autrice scrive:

Il Marocco degli europei - piatto, a compartimenti stagni, sovrapposizione di cartoline deformate dagli stereotipi e dall'ignoranza stessa degli osservatori - io l'ho rifiutato. Conoscerlo studiando, comparando e cercare di stabilire il nesso tra le sue diverse epoche, dimensioni e realtà mi è sempre parso l'unico modo per restituire a questo paese corpo e spessore. Per rendergli lo statuto di cultura. (Maraini 2000: 125) 
Il rifiuto di stereotipi di matrice orientalistica è, quindi, un fattore che accomuna Canetti e Maraini. Nel racconto dedicato a Marrakech «Ultimo tè a Marrakech», emergono altri elementi contenutistici che avvicinano le esperienze di viaggio dei due scrittori che hanno come meta la piazza di Djema el Fna e dintorni. La scrittrice italiana, come Canetti, fornisce una riflessione sulla città vecchia di Marrakech che va oltre lo spazio e il tempo. È un luogo che stuzzica quel che Maraini chiama «strati di memoria» (Maraini 2000: 78). Ciò spiega la confusione delle date, fornite dall'autrice, della visita ad un caffè nel vecchio Suq al-Sommariyn di Marrakech: «Eravamo nel 1965. O, forse, eravamo nel 1972 (o era il 1984?). A dire il vero, adesso che ci penso, in quel caffè sono rimasta seduta per più di vent'anni» (Maraini 2000: 78-79). Sorseggiare l'ultimo tè a Marrakech (esplicito è il riferimento al titolo della pellicola Ultimo tango a Parigi del regista italiano Bernardo Bertolucci) rappresenta, per la narratrice, un «momento sacro» (Maraini 2000: 81): un momento in cui si riuniscono elegantemente sapori (spezie e cannella), odori e le voci dei cantanti sufi.

In quest'atmosfera non può mancare altresì la figura del cantastorie, presentato dall'autrice come «giocoliere delle favole» e personificato da $\mathrm{H}$., protagonista del racconto e conoscente della narratrice. $\mathrm{H}$. è un profondo conoscitore di Marrakech: «Nessuno, proprio nessuno tra coloro che, locali e (estrema presunzione) stranieri, di lei hanno scritto, l'ha studiata e la conosce come H.» (Maraini 2000: 80). Quel che avvicina il personaggio H. a Maraini e, in un certo senso, a Canetti si potrebbe individuare nel rapporto nostalgico che li unisce alla città di Marrakech, con la differenza, ovviamente, che H. è originario di Marrakech. Il sentimento nostalgico provato dallo scrittore bulgaro e dall'autrice italiana in questo luogo è determinato dal loro anelito verso l'altrove, o meglio, dalla loro appartenenza extra-geografica all'altrove, quell'appartenenza che designa i confini spazio-temporali della memoria. Canetti, nascondendo le sue origini, scrive: «io ero quella piazza» (Canetti 2000: 57); e Maraini, la quale evita a più riprese di palesare la sua italianità, descrive così il proprio rapporto coi luoghi: «so che non appartengo più a nessun luogo e che alcuni luoghi mi appartengono per sempre» (Maraini 2000: 90) ${ }^{23}$.

Il riferimento che meglio interpreta il concetto di appartenenza, esposto dai due autori, è un celebre passo di Ugo di San Vittore (1069-1141), che troviamo in un'opera di Edward Said, l'intellettuale palestinese che ha vissuto la maggior parte della sua vita nell'altrove. Ecco le parole del teologo francese:

È dunque fonte di grande virtù per la mente educata apprendere, poco a poco, innanzitutto a passare attraverso le cose visibili e quelle transitorie, così che in seguito sia in grado di abbandonarle dentro di sé. L'uomo che trova dolce la propria terra è ancora un debole principiante; colui che considera ogni terra alla stregua di quella in cui è nato è già forte; ma perfetto è solo colui che al quale il mondo intero appare come una terra straniera. L'anima acerba concentra il suo 
amore su un posto nel mondo; l'uomo forte ha esteso il suo amore a ogni posto nel mondo; l'uomo perfetto l'ha saputo estinguere. (Said 2008: 229)

\section{BIBLIOGRAFIA}

Alighieri, A., «Opere minori», vol. I, Domenico de Robertis, Gianfranco Contini (a cura di), Vita Nuova. Rime, Milano, Riccardo Ricciardi Editore, 1995.

Bhabha, H., I luoghi della cultura, Roma, Meltemi, 2006.

Borghi, R., «Riflessioni sul senso del luogo. Il caso della Piazza Jamaa al Fna di Marrakech», Bollettino della Società geografica Italiana, (2005), pp. 1-17.

Bonu, D., «Die Verwandlung ist der Kernvorgang des Dramas. Note sulla concezione drammatica della letteratura di Elias Canetti», AnnalSS, 4, 2004 (2007), pp. 131-144. Caminati, L., Orientalismo eretico. Pier Paolo Pasolini e il cinema del terzo mondo, Milano, Bruno Mondadori, 2007.

Canetti, E., La lingua salvata. Storia di una giovinezza, Milano, Adelphi, 1991.

-, Le voci di Marrakech, Milano, Adelphi, 20001.

—, Il gioco degli occhi. Storia di una vita (1931-1937), Milano, Adelphi, 20002.

-, Massa e potere, Milano, Adelphi, 2002.

Chambers, I., Sulla soglia del mondo. L'altrove dell'occidente, Roma, Meltemi, 2003.

Ibn Arabi, M., L'interprete delle Passioni, Roberto Rossi Testa e Gianni De Martino (a cura di), Milano, Urra - Apogeo, 2008.

Lamri, T., I sessanta nomi dell'amore, Santarcangelo di Romagna, Fara Editore, 2006

Landau, R., Marrakech ba'd al-Istiklal (Marrakech dopo l'Indipendenza), traduzione di Khairi Hammad, Beirut, Dar al-Tali'a, 1961. Edizione originale: Landau, R., Morocco

- Independant, London, George Allen \& Unwin Ltd, 1961.

Laroui, A., Tarikhal-Maghreb (La storia del Maghreb), Al-mu'asasah al-Arabiya lil Dirasat wal Nashr, Beirut 1977. Edizione Originale: Laroui, A. L'histoire du Maghreb, La Découverte, 1970.

Lings, M., Che cos'è il Sufismo, Roma, Edizioni Mediterranee, 1978.

Loti, P., Les Désenchantées, Paris Calmann Lévy, 1906.

Maraini, T., Ultimo tè a Marrakech e nuovi racconti, Roma, Edizioni Lavoro, 2000.

Memmi, A., Portrait du colonise. portrait du colonisateur, Paris, Gallimard, 1985.

Nucera, D., «I viaggi e la letteratura», in Letteratura comparata, Armando Gnisci (a cura di), Milano, Bruno Mondadori, 2002.

Said, E., Orientalismo, traduzione di Stefano Galli, Milano, Feltrinelli, 1999. 
-, Nel segno dell'esilio. Riflessioni letture e altri saggi, traduzione di Massimiliano Guareschi e Federico Rahola, Milano, Feltrinelli, 2008. 
notAS Al FInAl

1 Il termine opedorico deriva dal greco hodós (via) e poréia (viaggio). Per il concetto di letteratura opedorica intesa come narrativa di viaggio si veda (Nucera 2002: 129-120).

2 A proposito di spazi inter-medi «in-between», Bhabha scrive: «Questi spazi inter-medi costituiscono il terreno per l'elaborazione di strategie del sé - come singoli o gruppo - che danno il via a nuovi segni di identità e luoghi innovativi in cui sviluppare la collaborazione e la contestazione nell'atto stesso in cui si definisce l'idea di società». (Bhabha 2006: 12).

3 Dai racconti de Le voci si comprende che la lingua francese è parlata a Marrakech soprattutto da chi ha ricevuto un'istruzione scolastica. Come si sa, la lingua francese ebbe una grande diffusione durante l'occupazione coloniale. Nonostante sia considerata dopo l'Indipendenza una lingua non ufficiale, viene tutt'ora utilizzata ampiamente nell'amministrazione e nei mass media. Per il concetto di bilinguismo coloniale nell'area nordafricana, si rimanda al celebre volume di Albert Memmi (Memmi 1985).

4 Un altro titolo che lascia riflettere sull'attenzione che l'autore bulgaro pone sulla lingua è quello del primo romanzo della sua trilogia autobiografica, La lingua salvata, (Die gerettete Zunge) uscito nel 1977.

5 Da notare che la ripetizione del nome di Allah ricorda il rituale mistico del sufismo conosciuto come dhikr. Si veda (Lings 1978: 78).

6 Non sono pochi gli scrittori europei ad essersi occupati dei segreti del mondo femminile in oriente. Tra i più celebri di questi è il romanziere francese Pierre Loti. Si veda Les Désenchantées (1906), il romanzo sulla vita privata delle donne turche.

7 Già nelle letterature medievali, araba e europea, vi è la tendenza a descrivere la presenza della donna angelicata come «un'apparizione». Si pensi alla donna persiana Nizam che ispira il poeta mistico andaluso Mu?yiddin Ibn Arabi in Turjuman al-Ashwaq (Ibn Arabi 2008). Seguendo il modello di Ibn Arabi, anche Dante Alighieri descrive Beatrice, in varie occasioni in Vita Nova, come «un miracolo» e «un'apparizione». Si veda (Alighieri 1995: 28-35). Non è da escludere che Canetti ebbe modo di approfondire questa tematica letteraria durante il suo soggiorno viennese. Nel suo romanzo autobiografico Il gioco degli occhi, Canetti rivela il fatto che il suo amico Dr. Sonne, profondo conoscitore della storia medievale, gli parlò del contatto della letteratura araba con quella europea medievale, aggiungendo: «da lui imparai non poco sulla poesia araba». (Canetti 20002: 341)

8 Canetti è esplicito su questo. Egli scrive a proposito delle grida dei medicanti ciechi: «Durante le settimane che ho trascorso in Marocco, non ho tentato di imparare né l'arabo né alcuna delle lingue berbere. Non volevo perdere nulla della forza di quelle strane grida». (Canetti 2000: 27).

9 Nella terminologia sufi, la parola fana' (estinzione) indica l'ultimo grado della certezza virtuale dell'anima verso la Verità divina. (Lings 1978: 59).

10 Trattando dei musulmani devoti, Canetti scrive: «I musulmani devoti si riuniscono in quattro diversi modi: $[\ldots]$ per la preghiera $[\ldots]$ per la guerra santa contro gli infedeli $[\ldots]$ alla Mecca, in occasione del grande pellegrinaggio. [...] nel giudizio universale». (Canetti 2002: 170).

11 Per le considerazioni metaletterarie nell'opera canettiana si veda, ad esempio, lo studio di Dina Bonu (Bonu 2007: 135).

12 Un esempio che illustra un incontro tra i cantastorie magrebini e i griot africani viene offerto dal racconto Il pellegrinaggio della voce, di Tahar Lamri. Lo scrittore italo-algerino affida la narrazione di un viaggio fantastico nel tempo e nello spazio ad un hakawati (cantastorie) proveniente da Marrakech dal nome Abdesslam El Sounbati detto El Marrakchi, il quale, con lo sviluppo degli eventi, cede la parola a Niang, un griot senegalese. È interessante notare come El Marrakchi si vanti di avere, nel suo percorso professionale, un'esperienza in Djema el Fna alle spalle. Nell'introduzione, egli recita: «Di professione e per vocazione cantastorie, ho cantato le lodi di Maometto, le prodezze di Alì e le gesta di Saad Zenati nella piazza di Jemaa El-Fena e in tutte le piazze d'Oriente». (Lamri, 2006: 90).

13 Canetti scrive nel capitolo dedicato al suk: «Non tutti [gli oggetti] sono belli, sempre di più s'intrufola tra loro robaccia di dubbia provenienza, fatta a macchina e importata dalle regioni del Nord. Ma il modo in cui sono presentati è ancora quello di una volta». (Canetti 2000: 23). 
14 La visione tipicamente orientalistica vuole che la parola «Oriente», scrive Said, sia «un sinonimo di stabilità e immutabile permanenza». (Said 1999: 237).

15 Per il concetto di «Terzo Mondo preistorico» nell'opera di Pasolini, si veda (Caminati 2007: 4). Il rapporto tra l'oriente e l'antichità spinge il regista friulano ad ambientare il suo celebre film Edipo re in Marocco, anziché Grecia.

16 Il numero corrisponde curiosamente ai quattordici capitoli che compongono Le voci di Marrakech.

17 Si noti che, tra tutti gli altri racconti, l'omonimo racconto, «Ultimo tè a Marrakech», è l'unico ambientato a Marrakech.

18 Si tratta della Festa del Sacrificio che viene celebrata dai musulmani ogni anno nel mese lunare di Dhu al-Hajj.

19 Lungi dal contesto narrativo, una testimonianza storica notevole che riguarda da vicino la posizione sociopolitica femminile marocchina è data dall'incarico attuale di Fatima Zahra Mansour, politica e avvocatessa marocchina, come primo sindaco donna di Marrakech.

20 È esemplare il rifiuto da parte di Agata, la protagonista del racconto «Una giornata, un fiume», di scrivere un articolo sul «conflitto tra tradizione e modernità», poiché «era stufa delle impasticciate diatribe sul Mediterraneo». (Maraini 2000: 23).

21 Per un'analisi approfondita sugli stereotipi nel contesto coloniale, si veda il capitolo «La questione dell'Altro. Stereotipo, discriminazione, e discorso del colonialismo» nel succitato libro di Homi Bhabha (Bhabha 2006: 97121).

22 La narratrice nel racconto «Viaggio a Thamusida» commenta a tal proposito: «La razionalità dell'Occidente è probabilmente soltanto un mito, come lo è l'irrazionalità supposta dell'Oriente» (Maraini 2000: 124).

23 In un'altra occasione, Maraini trova un'alternativa all'appartenenza di carattere geograficoidentitario. Scrive: «ho imparato a portare sedie, tavolini, libri, odori, musiche, pensieri, paesaggi, villaggi, porte - chiuse e aperte -, amici e nemici in una sola e unica valigia». (Maraini 2000: 95). 